\title{
Meropenem-induced Valproic Acid Elimination: A Case Report of Clinically Relevant Drug Interaction
}

\author{
Martin Šíma ${ }^{1}$, Jan Hartinger ${ }^{1}$, Jan Rulíšek ${ }^{2}$, Robert Šachl ${ }^{1,2}$, \\ Ondřej Slanar̆ \\ ${ }^{1}$ Institute of Pharmacology, First Faculty of Medicine, Charles University \\ and General University Hospital in Prague, Prague, Czech Republic; \\ ${ }^{2}$ Department of Anesthesiology and Intensive Care, First Faculty of Medicine, \\ Charles University and General University Hospital in Prague, Prague, Czech \\ Republic
}

Received April 24, 2017; Accepted August 28, 2017.

Key words: Valproic acid - Meropenem - Drug interaction - Therapeutic drug monitoring

\begin{abstract}
We present two case reports of drug interaction between valproic acid and meropenem. In comparison with expected population-kinetic based serum levels, we observed 90.8 and $\mathbf{9 3 . 5 \%}$ decrease in valproic acid serum levels during concomitant administration with meropenem. If carbapenems need to be administered to valproic acid treated patient, other anticonvulsant addition seems to be the appropriate as most probably the valproic acid dose escalation would not be sufficient to achieve therapeutic serum concentration.
\end{abstract}

This study was supported by the Charles University Project Progress Q25 and a grant No. SVV 260373.

Mailing Address: Martin Šíma, PharmDr., Institute of Pharmacology, First Faculty of Medicine, Charles University and General University Hospital in Prague, Albertov 4, 12800 Prague 2, Czech Republic; Phone: +420 224964 135; e-mail: martin.sima@If1.cuni.cz 


\section{Introduction}

Valproic acid (2-propylpentanoic acid,VPA) was introduced for clinical use in France in 1967. Over the next half-century it has become a well-established firstline and widely used agent for convulsion treatment both for its broad spectrum of activity against generalized and partial seizures and for its acceptable safety profile (Peterson and Naunton, 2005).VPA serum therapeutic range is $50-100 \mathrm{mg} / \mathrm{l}$ (346-693 $\mu \mathrm{mol} / \mathrm{l})$ in seizure treatment (Bentué-Ferrer et al., 2010). Although VPA therapeutic drug monitoring is not routinely recommended in common neurological practice (Tomson et al., 2007) it can be useful in polypharmacy to reveal possible drug interactions or in the intensive care units when VPA is initiated for the onset of seizure treatment (Loh et al., 2010).

There are at least three metabolic pathways of VPA elimination in humans: glucuronidation, mitochondrial beta-oxidation (both considered major routes accounting for 50 and $40 \%$ of dose, respectively) and cytochrome P450-mediated oxidation (minor route responsible for elimination of approximately $10 \%$ of dose) (Ghodke-Puranik et al., 2013). Concurrently, VPA is regenerated from VPA-glucuronide by acylpeptide hydrolase-mediated hydrolysis. Carbapenems inhibit acylpeptide hydrolase and thus VPA regeneration from VPA-glucuronide, resulting in reduced VPA serum levels (Nakajima et al., 2004; Suzuki et al., 2010, 2011).

There are few case reports describing decreased VPA serum levels by carbapenems (Nacarkucuk et al., 2004; Clause et al., 2005; Fudio et al., 2006; Suntimaleeworakul et al., 2012). A recent study also documented the decreased VPA levels caused by concomitant use of meropenem using a large number of therapeutic drug monitoring (TDM) records (Wen et al., 2017). We present additional two cases of this significant drug interaction.

\section{Case report 1}

A 59-year-old polymorbid male $(90 \mathrm{~kg}, 180 \mathrm{~cm})$ admitted to the Department of Anesthesiology and Intensive Care after cardiopulmonary resuscitation for cardiorespiratory arrest. The sixth day of hospital stay VPA treatment was initiated for generalized myoclonic seizures probably related to brain ischemia. Patient was loaded with 1,600 mg VPA administered via $24 \mathrm{~h}$-continuous intravenous infusion, followed by maintenance doses of $500 \mathrm{mg}$ given every 8 hours as crushed slowrelease tablets through the nasogastric tube. The tenth day of hospital stay meropenem therapy was initiated $(2 \mathrm{~g}$ intravenous bolus followed by $4 \mathrm{~g} /$ day in continuous intravenous infusion) for pulmonary infection. The other concomitant medication included norepinephrine, unfractionated heparin (UFH), insulin, propofol, potassium supplementation, omeprazole, prednisone, diosmectite and probiotics. After five days of concomitant VPA and meropenem administration, blood sample was taken, showing a serum level of VPA of $8.0 \mathrm{mg} / \mathrm{l}$ (determined using a cloned enzyme donor immunoassay with quantification limit of $3 \mathrm{mg} / \mathrm{l})$. In 
spite of the low VPA levels no other seizures episode occurred. Subsequently VPA treatment was discontinued.

\section{Case report 2}

A 56-year-old man $(92 \mathrm{~kg}, 178 \mathrm{~cm})$ with chronic thrombotic pulmonary hypertension was admitted to the surgical ward for elective pulmonary vein endarterectomy. Patients' medical history included congestive hepatopathy, history of minor stroke, mild chronic kidney disease, dyslipidemia, hypertension and schizophrenia. On the second postoperative day after successful surgery intravenous meropenem $2 \mathrm{~g}$ three times a day was initiated because of pulmonary infection. As generalized myoclonic seizures occurred the day after, VPA treatment was initiated. Intravenous loading dose of $1,200 \mathrm{mg}$ was followed by $100 \mathrm{mg} / \mathrm{hour}$ continuous infusion for 24 hours. Subsequently the patient was treated with maintenance doses of $300 \mathrm{mg}$ every 8 hours administered as crushed slow release tablets through the nasogastric tube. On the postoperative day 7 the trough level of VPA was $3.4 \mathrm{mg} / \mathrm{l}$ (determined using cloned enzyme donor immunoassay). Concomitant medication included norepinephrine, UFH, insulin, propofol, sufentanyl, potassium supplementation, vancomycin, acetaminophen, dipyrone, omeprazole, furosemide, risperidone and treprostinil. In spite of the low VPA levels no other seizures episode occurred through the rest of hospital stay. Meropenem was withdrawn on postoperative day 8 and VPA two weeks later shortly before the hospital discharge.

\section{Discussion}

Food and Drug Administration Professional Drug Information (FDA-PDI) states following mean VPA population pharmacokinetic parameters: volume of
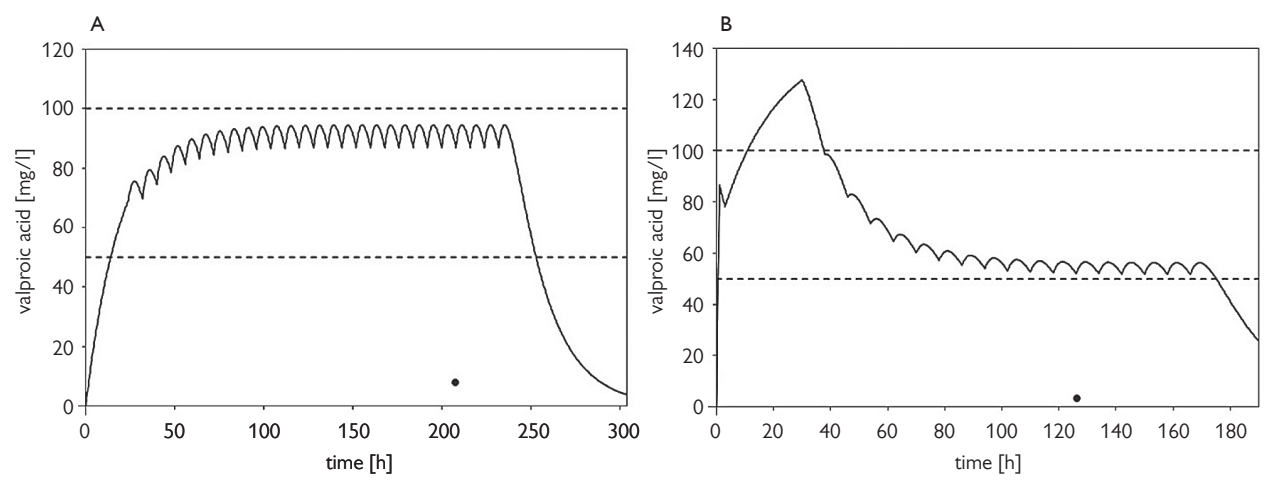

Figure 1 -A priori simulation of valproic acid plasma levels time profile based on population pharmacokinetics compared with measured valproic acid concentration. A) case report 1;B) case report 2. Dotted lines represent therapeutic range. 
distribution $11 \mathrm{l} / 1.73 \mathrm{~m}^{2}$, clearance $0.56 \mathrm{l} / \mathrm{h} / 1.73 \mathrm{~m}^{2}$ and terminal elimination half-life $\left(T_{1 / 2}\right) 16 \mathrm{~h}$. Simulation of VPA serum levels based on FDA-PDI population pharmacokinetics compared with measured VPA concentration is shown in Figure 1. Pharmacokinetic models show that measured VPA serum concentrations corresponded to only 9.2 and $6.5 \%$ of concentrations estimated based on population data. Fitting of the simulated pharmacokinetic profile curve with observed drug concentration points resulted in VPA $T_{1 / 2}$ of 1.6 and $1.4 \mathrm{~h}$. Very short $T_{1 / 2}$ indicates that VPA dose escalation is meaningless in this case, while switch to another antiepileptic agent seems to be more relevant for effective anticonvulsive treatment.

It should be noted, however, that in theory the case 1 VPA levels could be also affected by reduced VPA absorption due to concomitant diosmectite administration. However, diosmectite was administered in at least $2 \mathrm{~h}$ distance from the other medication and no adsorbent was administered to the patient in case 2 where comparably low concentration of VPA was measured. In both cases slow release tablets were crushed to allow administration through nasogastric tube. Nevertheless nasogastric tube should have negligible impact. VPA bioavailability is approaching $100 \%$ when immediate release form is used and 8 hour dosing interval would not result in as low serum trough levels as were measured if clearance of the drug was not affected. Based on the literature data VPA-meropenem interaction is the most probable explanation for this observation. As absorption extent and volume of distribution is likely not to be affected by concomitant meropenem administration, significant increase in VPA clearance due to inhibition of VPA-glucuronide cleavage is most probable mechanism of this interaction.

Carbapenems are known for seizure threshold lowering properties in comparison with non-carbapenem antibiotics. Even though this adverse event was described mostly for imipenem/cilastatin, head to head comparison with meropenem showed similar risks for imipenem and meropenem (Cannon et al., 2014). In case 2, possible contribution of meropenem in triggering seizures cannot be excluded. Considering carbapenem-VPA interaction, appropriate treatment of suspected carbapenem-induced seizures should not include VPA.

\section{Conclusion}

We present two case reports of drug interaction between VPA and meropenem. In comparison with expected population-kinetic based serum levels, we observed 90.8 and $93.5 \%$ decrease in VPA serum levels during concomitant administration with meropenem. It is important for the physicians to recognize drug interaction between VPA and carbapenems, especially when treating carbapenem induced seizures. If carbapenems need to be administered to VPA treated patient other anticonvulsant addition seems to be appropriate as VPA dose escalation would most probably not be sufficient to achieve therapeutic serum concentration. 


\section{References}

Bentué-Ferrer, D., Tribut, O., Verdier, M. C., Suivi Thérapeutique Pharmacologique de la Société Française de Pharmacologie et de Thérapeutique (2010) Therapeutic drug monitoring of valproate. Therapie 65, 233-240. (in French)

Cannon, J. P., Lee, T. A., Clark, N. M., Setlak, P., Grim, S. A. (2014) The risk of seizures among the carbapenems: a meta-analysis. J. Antimicrob. Chemother. 69, 2043-2055.

Clause, D., Decleire, P.Y., Vanbinst, R., Soyer, A., Hantson, P. (2005) Pharmacokinetic interaction between valproic acid and meropenem. Intensive Care Med. 31, 1293-1294.

Fudio, S., Carcas, A., Piñana, E., Ortega, R. (2006) Epileptic seizures caused by low valproic acid levels from an interaction with meropenem. J. Clin. Pharm. Ther. 31, 393-396.

Ghodke-Puranik, Y., Thorn, C. F., Lamba, J. K., Leeder, J. S., Song, W., Birnbaum, A. K., Altman, R. B., Klein, T. E. (2013) Valproic acid pathway: pharmacokinetics and pharmacodynamics. Pharmacogenet. Genomics 23, 236-241.

Loh, G.W., Mabasa, V. H., Ensom, M. H. (2010) Therapeutic drug monitoring in the neurocritical care unit. Curr. Opin. Crit. Care 16, 128-135.

Nacarkucuk, E., Saglam, H., Okan, M. (2004) Meropenem decreases serum level of valproic acid. Pediatr. Neurol. 31, 232-234.

Nakajima, Y., Mizobuchi, M., Nakamura, M., Takagi, H., Inagaki, H., Kominami, G., Koike, M., Yamaguchi, T. (2004) Mechanism of the drug interaction between valproic acid and carbapenem antibiotics in monkeys and rats. Drug Metab. Dispos. 32, 1383-1391.

Peterson, G. M., Naunton, M. (2005) Valproate: a simple chemical with so much to offer. J. Clin. Pharm. Ther. 30, 417-421.

Suntimaleeworakul,W., Patharachayakul, S., Chusri, S. (2012) Drug interaction between valproic acid and meropenem: a case report. J. Med. Assoc. Thai. 95, 293-295.

Suzuki, E., Yamamura, N., Ogura, Y., Nakai, D., Kubota, K., Kobayashi, N., Miura, S., Okazaki, O. (2010) Identification of valproic acid glucuronide hydrolase as a key enzyme for the interaction of valproic acid with carbapenem antibiotics. Drug Metab. Dispos. 38, 1538-1544.

Suzuki, E., Nakai, D., Yamamura, N., Kobayashi, N., Okazaki, O., Izumi, T. (2011) Inhibition mechanism of carbapenem antibiotics on acylpeptide hydrolase, a key enzyme in the interaction with valproic acid. Xenobiotica 41, 958-963.

Tomson, T., Dahl, M. L., Kimland, E. (2007) Therapeutic monitoring of antiepileptic drugs for epilepsy. Cochrane Database Syst. Rev. 2, CD002216.

Wen, Z. P., Fan, S. S., Du, C., Yin, T., Zhou, B. T., Peng, Z. F., Xie, Y.Y., Zhang, W., Chen, Y., Xiao, J., Chen, X. P. (2017) Drug-drug interaction between valproic acid and meropenem: a retrospective analysis of electronic medical records from neurosurgery inpatients. J. Clin. Pharm. Ther. 42, 221-227. 